\title{
Enhancement of piezoelectric properties stability of submicron- structured piezoceramics obtained by spark plasma sintering
}

\author{
Diego A. Ochoa, ${ }^{1}$ Francesc Suñol, ${ }^{1}$ Fernando Rubio-Marcos, ${ }^{2}$ and Jose E. García ${ }^{1,}$ \\ ${ }^{1}$ Department of Physics, Universitat Politècnica de Catalunya - BarcelonaTech, 08034 Barcelona, Spain. \\ ${ }^{2}$ Department of Electroceramic, Instituto de Cerámica y Vidrio, CSIC, 28049 Madrid, Spain.
}

\begin{abstract}
Grain-size effect on functional properties of piezoceramics is nowadays an important topic due to the potential applications of these materials at the submicron scale. In this context, we explore the effect of the grain size reduction on the functional properties stability of piezoceramics. Submicron- and micro-structured $\mathrm{Pb}(\mathrm{Zr}, \mathrm{Ti}) \mathrm{O}_{3}$-based materials are taken as model samples to carry out this study. Rayleigh analysis is used to quantitatively evaluate the domain wall contribution. The results show that the stability of properties depends strongly on grain size. Specifically, the nonlinear response decreases significantly when the grain size is reduced to the submicron scale. This behavior can be explained by taking into account that a grain size reduction involves a grain boundary increment, which may clamp domain walls. The results suggest that grain size engineering may be an effective alternative to the widely used compositional engineering in order to control the undesirable nonlinear behavior in piezoceramics.
\end{abstract}

Keywords: piezoceramics; grain-size effect; nonlinear response; domain wall motion; Rayleigh behaviour

* Corresponding author.

E-mail address: jose.eduardo.garcia@upc.edu (J.E. García). 


\section{Introduction}

Nanostructured materials fascinate the scientific community because of their novel properties and applications. Belonging to these attractive materials are nanostructured piezoceramics, which are usually three-dimensional solids composed of nanometer-sized grains. The thickness of the piezoceramic layers need to be reduced down to the micrometer for their integration into novel ceramic technologies, thereby requiring a decrease in grain size down to the submicron range. Confining the grain size close to the nanoscale entails remarkable functional properties of piezoceramics that could be exploited [1-3].

The performance of piezoelectric ceramics is closely related to microstructure, and consequently to the sintering method. Solid state reaction is considered the conventional sintering (CS) of oxide-based electroceramics, which results in dense materials. However, although dense piezoceramics can be easily obtained through CS, the final grain size is above $\sim 0.5$ um, even when using nanometer-sized powder. Many rapid sintering techniques have been developed over the last decades in order to obtain nanostructured ceramics, among which spark plasma sintering (SPS) has been widely used to obtain piezoceramics with grain sizes of approximately $\sim 100 \mathrm{~nm}$ or even lower $[4,5]$.

It is well known that the macroscopic functional properties differences between nano-, submicron-, and micro-structured piezoceramics is linked to how the grain size scales the properties [6-11]. This leads to the assumption that a grain-size dependence on the nonlinear response of piezoceramics must exist, given the understanding of nonlinear response as the variation of the functional properties under an external stimulus; i.e., the stability of properties versus electric fields or mechanical stresses. Taking into account that the nonlinear behavior is a drawback of piezoelectric materials for many applications (especially when piezoelectric 
materials work under pressure), it is vital to gain an understanding of how such nonlinear behavior is modified by the decrease in grain size.

The nonlinear response of piezoceramics are addressed in terms of two contributions known as intrinsic (that is, the contribution of composition, crystal structure, and so on) and extrinsic (that is, the contribution of grain size, ferroelectric domain configuration, domain wall dynamics, and so on) contributions [12]. However, the main contribution to the piezoelectric response of piezoceramics at room temperature is the extrinsic contribution [12-16]. Given that the extrinsic contribution is grain-size dependent, a significant decrease in grain size is expected to produce a notable modification of the nonlinear response of piezoceramics. Thus, the stability of the piezoelectric properties of these materials may be improved by controlling the grain size.

Although the grain-size effect on the macroscopic functional properties of piezoceramics has been widely studied, very few works correlate grain size and properties stability. This paper is therefore focused on exploring the effect of a grain-size reduction in the nonlinear dielectric and piezoelectric response of piezoceramics. To achieve this goal, we build on the previous knowledge acquired about the nonlinear response of the $\mathrm{Pb}(\mathrm{Zr}, \mathrm{Ti}) \mathrm{O}_{3}$-based piezoceramics system [17-19]. Nb-doped, morphotropic phase boundary, $\mathrm{Pb}(\mathrm{Zr}, \mathrm{Ti}) \mathrm{O}_{3}$ samples, obtained from the same powder but by different sintering methods (CS and SPS), are taken as model materials on which to base the study. The results show that the stability of functional properties of piezoceramics is very closely related to grain size.

\section{Experimental}

\subsection{Samples preparation}

A conventional oxide mixing process was used to prepare $\mathrm{Pb}\left(\mathrm{Zr}_{0.53} \mathrm{Ti}_{0.47}\right) \mathrm{O}_{3}$ nanopowders. The composition was selected to exhibit morphotropic phase boundary, a compositional-driven boundary where the functional properties of piezoelectrics maximize as a consequence of 
intrinsic and extrinsic contribution enhancement [20,21]. In order to obtain an appropriate distribution of the particle size, the starting raw materials (i.e. $\mathrm{PbO}, \mathrm{ZrO}_{2}$ and $\mathrm{TiO}_{2}$ ) were individually milled by attrition milling in ethanol for $3 \mathrm{~h}$. These powders were then stoichiometrically weighed and mixed by attrition milling using $\mathrm{ZrO}_{2}$ balls in ethanol medium for $3 \mathrm{~h}$, dried at $80{ }^{\circ} \mathrm{C}$ and calcined at $850{ }^{\circ} \mathrm{C}$ for $3.5 \mathrm{~h}$. To design a soft-type piezoceramics material, $1 \mathrm{wt} . \%$ of $\mathrm{Nb}_{2} \mathrm{O}_{5}$ was added to the green moisture before homogenization by a second ball milling, thereby obtaining a Nb-doped PZT composition, hereafter labelled as PNZT.

In order to evaluate how grain size impacts on the piezoelectric properties stability, we conducted the sintering process according to two methods: conventional sintering (CS) and spark plasma sintering (SPS). For the first approach (that is, CS), the calcined powders were uniaxially pressed at $100 \mathrm{MPa}$ into pellets of $20 \mathrm{~mm}$ in diameter and approximately $5 \mathrm{~mm}$ in length, which were then isostatically pressed at $200 \mathrm{MPa}$. The pellets were then sintered in air at $1250^{\circ} \mathrm{C}$ for 330 min in a saturated $\mathrm{PbO}$ atmosphere. The heating rate was maintained $3{ }^{\circ} \mathrm{C} / \mathrm{min}$, and the cooling rate followed the furnace cooling. By contrast, the SPS consolidation was performed using a system DR SINTER SPS-1050-CE, which enabled fine-grained PNZT ceramics to be prepared. In this case, the pellet was held in a graphite die with an inside diameter of $20 \mathrm{~mm}$ and approximately $5 \mathrm{~mm}$ in length at a heating rate of $120{ }^{\circ} \mathrm{C} / \mathrm{min}$, an axial pressure of $50 \mathrm{MPa}$ and a holding time of $1 \mathrm{~min}$ at $1000{ }^{\circ} \mathrm{C}$. Sintered PNZT-CS and PNZT-SPS samples were cut into disks of $15 \mathrm{~mm}$ in diameter and $0.8 \mathrm{~mm}$ in thickness, avoiding $\mathrm{Pb}$ inhomogeneous areas. Gold electrodes were sputtered on both faces of the samples to perform the electrical characterizations.

\subsection{Structure and microstructure}

$\mathrm{X}$-ray diffraction (XRD) measurements were performed using the Bragg-Brentano configuration with a Bruker AXS D8 Advance diffractometer $(\mathrm{CuK} \alpha$ radiation $\lambda=1.5418 \AA$ ). Based on the XRD analysis, both XRD diffraction patterns show the stabilization of the MPB region (Fig. 
This is the post-print (i.e. final draft post-refereeing) of the publication.

The final publication is available at Elsevier via http://dx.doi.org/10.1016/j.jeurceramsoc.2018.06.016

1(a)-(b)), which is associated with the room temperature coexistence between a tetragonal symmetry $(\mathrm{P} 4 \mathrm{~mm})$, and a rhombohedral symmetry $(\mathrm{R} 3 \mathrm{~m})$. One may observe that the diffraction peaks are in the same two-theta positions, thereby showing that sintering methods have no significant effect on the crystallographic phase. The microstructure was evaluated on polished and thermally etched samples using a Veeco multimode Atomic Force Microscope (AFM) with a Nanoscope IIIA. As expected, the topographic characterization (Fig. 1(c)-(d)) shows dense ceramics with a significant difference in the average grain size. Thus, the grain size distribution analysis (Fig. 1(e)-(f)) shows an average grain size of $\sim 3.4 \mu \mathrm{m}$ for PNZT-CS and $\sim 330 \mathrm{~nm}$ for

\section{PNZT-SPS.}

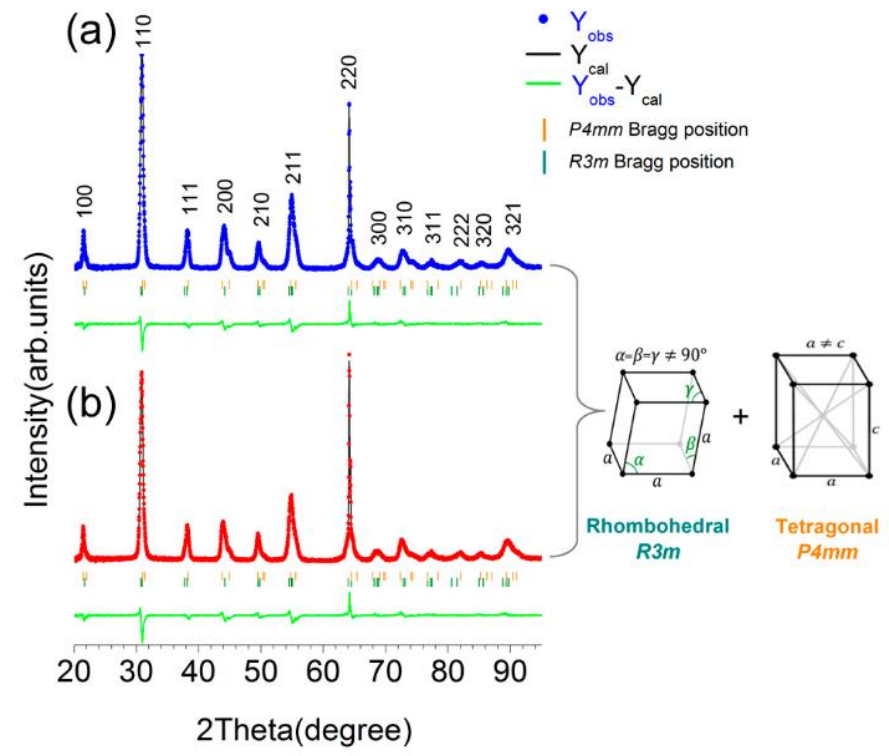

(c)

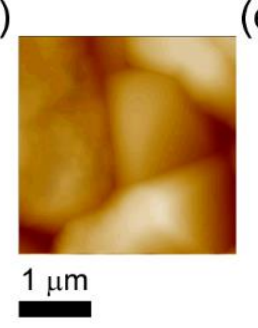

(d)

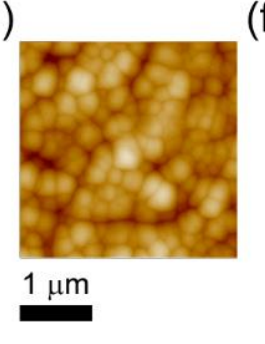

(e)

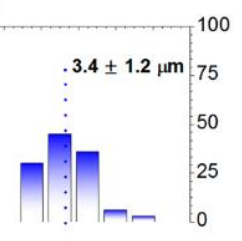

(f)

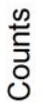

Fig. 1. Structural and microstructural characterization: X-ray diffraction pattern for Nb-doped $\mathrm{Pb}\left(\mathrm{Zr}_{0.53} \mathrm{Ti}_{0.47}\right) \mathrm{O}_{3}$ obtained by (a) conventional sintering (CS) or (b) spark plasma sintering (SPS). Additionally, a schematic phase coexistence between a tetragonal symmetry (P4mm), and a rhombohedral symmetry (R3m) is shown on the right of the panels (a) and (b). Topography images are shown in (c) for $\mathrm{CS}$ and (d) for SPS. Note that cross sections of each sample were polished and then thermally etched at $1050{ }^{\circ} \mathrm{C}$ and $850{ }^{\circ} \mathrm{C}$ (that is, a $15 \%$ less than the sintering temperature) for 5 minutes, respectively. The grain size distribution analysis is reported in (e) for CS and (f) for SPS. 


\subsection{Electrical characterization}

The temperature dependence of the dielectric response was measured using a precision LCR meter (Agilent E4980A). The samples were placed in a closed loop cryogenic system consisting of a helium compressor (Cryogenics 8200), a cold finger (Cryogenic model 22) and a vacuum pump (Alcatel Drytel Micro CFV100D). The system enabled a sweep in a temperature range from $30 \mathrm{~K}$ to $390 \mathrm{~K}$ by using a temperature controller (LakeShore model 331). The permittivity dependence with a sub-switching ac electric field was measured at $1 \mathrm{kHz}$ by means of a capacitance comparator bridge especially designed for this type of measurement. The instantaneous nonlinear current was measured and taken as a function of the instantaneous applied electric field. For each value of the voltage amplitude, the electrical displacement was obtained by numerical integration of the current. The values of the real and imaginary parts of the permittivity were computed from the components of the dielectric response in phase and in quadrature of phase with the applied field (details on this measurement are given in [22]). Electric field-induced polarization hysteresis loops were measured in a typical Sawyer-Tower configuration by applying a sinusoidal electric field of amplitude of $3.0 \mathrm{kV} / \mathrm{mm}$ at a frequency of $1 \mathrm{~Hz}$, using a Hewlett-Packard 33120A signal generator and a Trek 663 signal amplifier. The electric field-induced macroscopic strain was measured by adding a WayCon inductive position transducer conditioning with a Solartron OD5 Module to the previous setup.

Nonlinear longitudinal piezoelectric responses (dynamic piezoelectric coefficient) were measured by a Berlincourt-type method. A sinusoidal stress, with variable amplitude and frequency of $1 \mathrm{~Hz}$, was applied to the samples superimposed to a constant compression force (details on this measurement are given in [23]). The compression force did not exceed $60 \mathrm{MPa}$ in order to avoid irreversible depoling of the samples, which was checked after piezoelectric measurement by the resonant method. The static direct piezoelectric characterization was 
performed using a KFC Technologies PM3500 $\mathrm{d}_{33}$ meter (i.e., the classic Berlincourt measurement).

\section{Results and discussions}

\subsection{Dielectric, ferroelectric and electromechanical responses}

Fig. 2 shows the strain- and polarization-electric field $(S-E$ and $P-E$ ) hysteresis loops for both PNZT-CS and PNZT-SPS samples. Some interesting features may be observed. On the one hand, the electric field-induced strain as well as the spontaneous strain are clearly higher in PNZT-CS, although the mechanical response of PNZT-SPS is not poor. A typical $S$ - $E$ loop corresponding to a soft piezoelectric material is displayed for PNZT-CS, whereas a flattened loop is shown for PNZT-SPS, which suggests a certain hardening related to grain size reduction. On the other hand, the ferroelectric response shows almost the same remnant polarization for both samples, while PNZT-SPS shows a higher coercive field. The increase in the coercive field as a result of grain size reduction has been reported for various perovskite piezoceramic systems, as a consequence of the domain wall pinning effect by decreasing the domain size. In summary, only a slight hardening seems to be induced by grain-size reduction up to submicron scale. In fact, remnant polarization remains unaltered, thereby suggesting a possible good piezoelectric performance for submicron structured materials. 


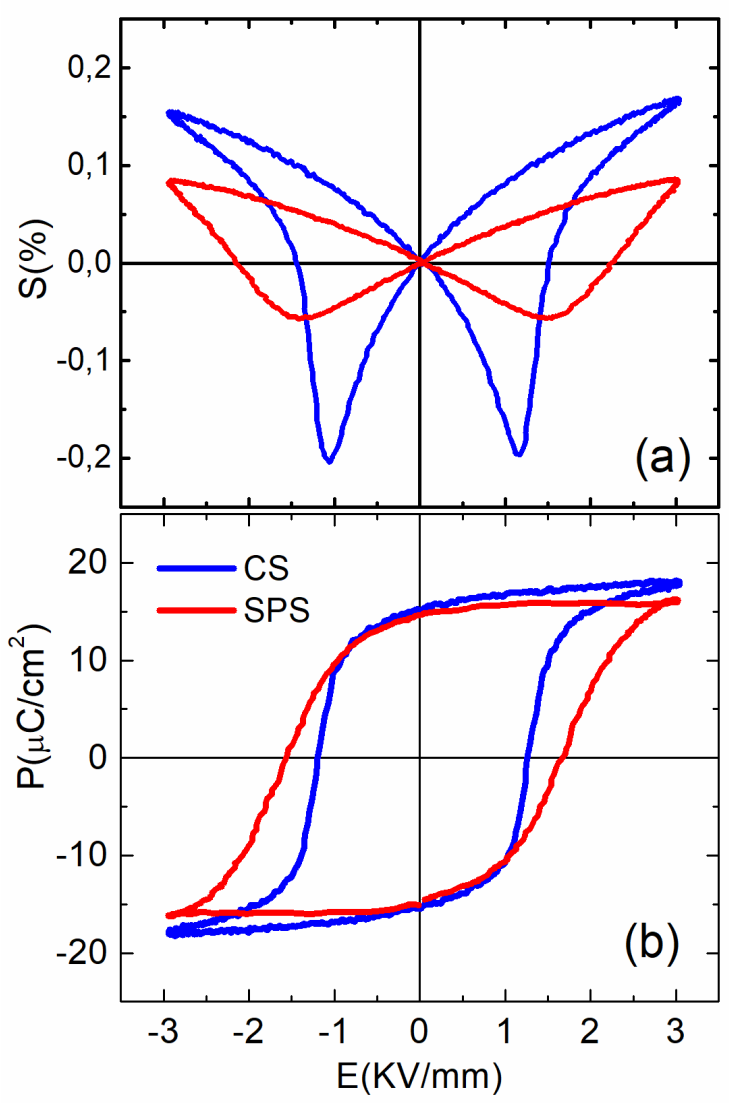

Fig. 2. Ferroelectric and electromechanical responses: (a) Strain-electric field ( $S-E)$ and (b) polarizationelectric field $(P-E)$ hysteresis loops for $\mathrm{Nb}$-doped $\mathrm{Pb}\left(\mathrm{Zr}_{0.53} \mathrm{Ti}_{0.47}\right) \mathrm{O}_{3}$ obtained by conventional sintering (CS) or spark plasma sintering (SPS), measured at $1 \mathrm{~Hz}$ and at room temperature.

Fig. 3 shows the temperature dependence of the dielectric response, $\varepsilon^{\prime}(T)$, from low to room temperature, for both PNZT-CS and PNZT-SPS samples. As may be observed, the values of $\varepsilon$ ' at very low temperatures are almost the same for both samples, thereby suggesting the same intrinsic dielectric response (i.e., the value of $\varepsilon^{\prime}(T=0)$ ). This is in agreement with the fact that only extrinsic contributions are altered by the reduction in grain size. Recovering the $\varepsilon^{\prime}(T)$ curves, a monotonically increase of $\varepsilon$ ' with increasing temperature for both samples is shown, which is a typical feature for soft PZTs materials. However, the PNZT-CS response at room temperature is $30 \%$ higher than PNZT-SPS. Taking into account that the intrinsic contribution does not change with the increase in temperature [12], it is possible to assume that the difference 
in the dielectric response at room temperature is due to different extrinsic contributions related to a grain size effect.

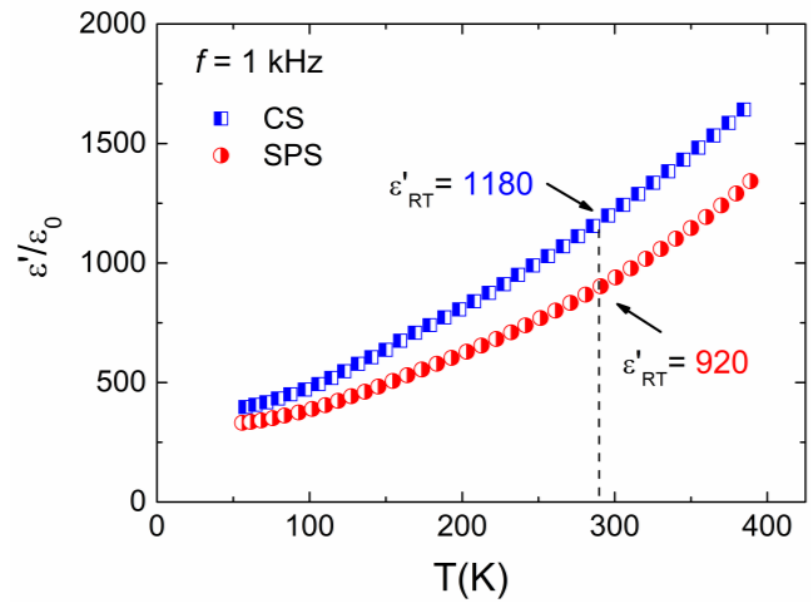

Fig. 3. Temperature dependence of dielectric constant from low temperature to above room temperature for $\mathrm{Nb}$-doped $\mathrm{Pb}\left(\mathrm{Zr}_{0.53} \mathrm{Ti}_{0.47}\right) \mathrm{O}_{3}$ obtained by conventional sintering (CS) or spark plasma sintering (SPS). The values of the room temperature dielectric constants are indicated in the plot.

\subsection{Nonlinear dielectric response}

Nonlinear measurements have emerged as a powerful experimental tool for characterizing the domain wall motion and domain wall dynamics in piezoelectric materials [24,25]. The increment in the real and imaginary parts of the dielectric coefficient ( $\varepsilon^{\prime}$ and $\varepsilon '$ ') with the amplitude of the applied electric field $\left(E_{0}\right)$ is shown in Fig. 4. As may be observed, PNZT-CS and PNZT-SPS nonlinear responses are qualitatively equal; that is, $\varepsilon$ ' and $\varepsilon$ ' increase quasi-linearly with increasing $E_{0}$. Nevertheless, quantitative differences exist (i.e., different slopes for $\varepsilon^{\prime}$ and $\varepsilon^{\prime \prime}$ versus $E_{0}$ curves) that suggest differences in the domain wall dynamics and/or domain wall motion. 


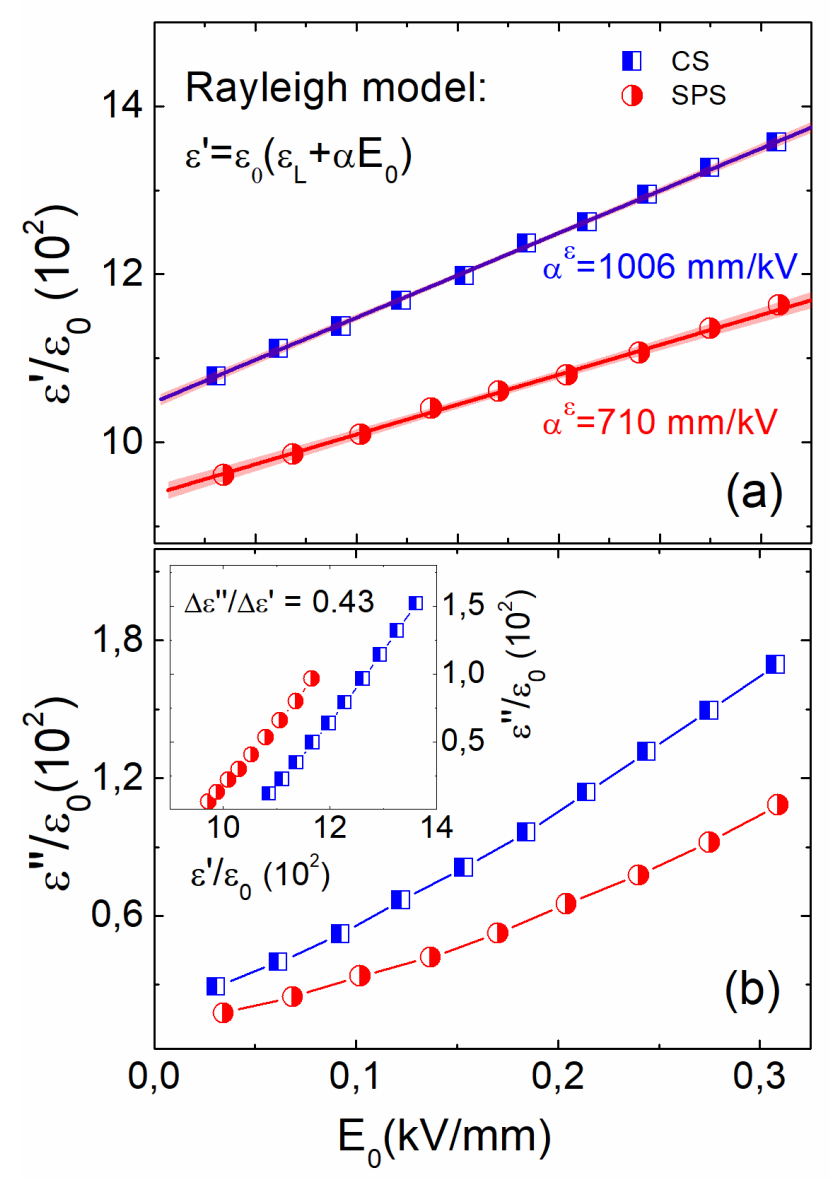

Fig. 4. Nonlinear dielectric response: real (a) and imaginary (b) parts of the relative permittivity as a function of the amplitude of the applied electric field for Nb-doped $\mathrm{Pb}\left(\mathrm{Zr}_{0.53} \mathrm{Ti}_{0.47}\right) \mathrm{O}_{3}$ obtained by conventional sintering (CS) or spark plasma sintering (SPS), measured at $1 \mathrm{kHz}$ and at room temperature. Relation between real and imaginary parts of the relative permittivity are shown in the inset.

A quantitative study of the nonlinear response can be carried out by using the Rayleigh model [24-27]. The main hypothesis underpinning the Rayleigh model is that the material response is exclusively due to the extrinsic contribution related to irreversible motion of the ferroelectric domain walls [26]. According to this model, the variation of $\varepsilon$ ' and $\varepsilon$ ' with $E_{0}$ follow a linear dependence that can be written as [27]:

$$
\begin{aligned}
& \varepsilon^{\prime}=\varepsilon_{0}\left(\varepsilon_{L}+\alpha^{\varepsilon} E_{0}\right) \\
& \varepsilon^{\prime \prime}=\varepsilon_{0}\left(\frac{4}{3 \pi} \alpha^{\varepsilon} E_{0}\right)
\end{aligned}
$$


where $\alpha^{\varepsilon}$ is the Rayleigh coefficient and $\varepsilon_{L}$ is the dielectric coefficient at zero-field amplitude (i.e. the commonly reported dielectric constant, as $\varepsilon^{\prime}{ }_{\mathrm{RT}}$ in Fig. 3). Additionally, in order to fulfill the requirements of the model, the ratio of the increments $m_{\varepsilon}=\frac{\Delta \varepsilon^{\prime \prime}}{\Delta \varepsilon^{\prime}}=\frac{4}{3 \pi} \cong 0.42$ should be a constant value. The inset in Fig. 4 show a value of $m_{\varepsilon}=0.43$, proving that for these samples the model is fulfilled.

Once the requirements of the Rayleigh model have been met, $\alpha^{\varepsilon}$ becomes a useful parameter for quantifying the stability of the dielectric response. The $\alpha^{\varepsilon}$ value is easily obtained by a linear fitting of $\varepsilon^{\prime}$ versus $E_{0}$ curve, as shown in Fig. 4(a). By comparing the values of $\alpha^{\varepsilon}$, it is possible to affirm that the nonlinear response is significantly lower for PNZT-SPS. This is a remarkable result, since it constitutes an enhancement of dielectric properties stability by gran-size reduction, which could be translated into applications. The grain-size effect on the nonlinear response can be explained by taking into account that a grain-size reduction involves a grain boundary increment. The grain boundaries clamp domain walls, thereby making their motion more difficult.

\subsection{Direct piezoelectric response}

The observed stability enhancement of dielectric properties by grain-size reduction suggests that an improvement of piezoelectric properties stability may also be achieved, since a common origin of the nonlinear response, either dielectric or piezoelectric, is expected from perovskite piezoceramics [28]. From this perspective, Fig. 5(a) shows the dependence of the longitudinal piezoelectric coefficient $d_{33}$ with the amplitude $T_{0}$ of the applied dynamical stress for a given 'bias' uniaxial compressive stress $T_{\mathrm{DC}}$. Although the characterization was performed at different pre-stress compressions, a $T_{\mathrm{DC}}=40 \mathrm{MPa}$ was chosen for the quantitative study because it is a 
representative value at which the linear response $d_{330}$ is the same for both samples, as shown in Fig. 5(b). PNZT-CS shows a large nonlinear piezoelectric response $\Delta d_{33}=69 \mathrm{pC} / \mathrm{N}$ (35\% of the initial value) at $T_{0}=9 \mathrm{MPa}$ in concordance with previous works [29], while PNZT-SPS shows a moderate nonlinear response $\Delta d_{33}=22 \mathrm{pC} / \mathrm{N}\left(12 \%\right.$ of the initial value) at the same $T_{0}$.

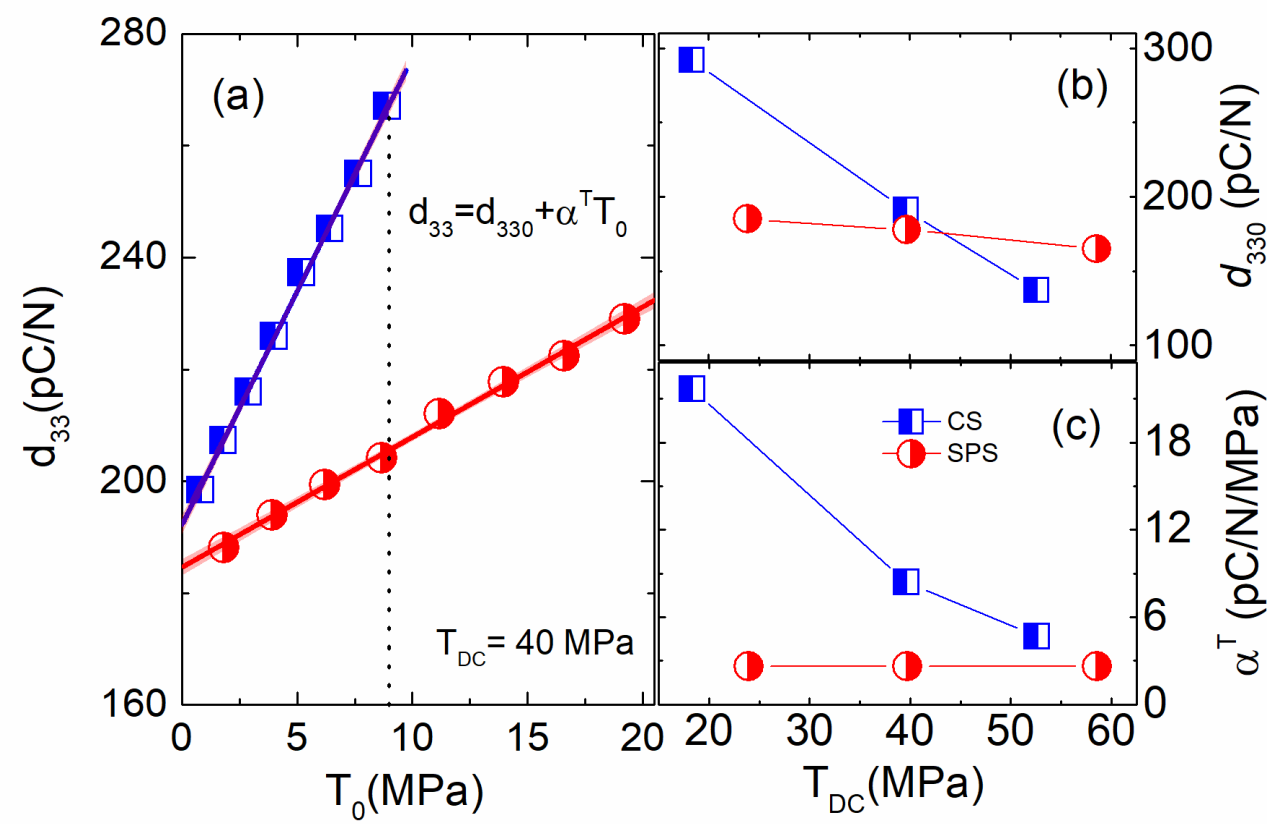

Fig. 5. Piezoelectric response: (a) direct piezoelectric coefficient $d_{33}$ as a function of the amplitude of the applied dynamical stress $T_{0}$, measured at $1 \mathrm{~Hz}$ and at room temperature. In panel (b), $d_{330}$ represents the direct piezoelectric coefficient at zero-stress amplitude $\left(T_{0}=0\right)$, which is plotted as a function of the uniaxial compression pre-stress, $T_{\mathrm{DC}}$. The Rayleigh coefficient $\alpha^{\mathrm{T}}$ is obtained by a linear fitting of the $d_{33}$ versus $T_{0}$ curves and is plotted in panel (c) as a function of $T_{\mathrm{DC}}$.

Using the Rayleigh model equation to quantify the nonlinear direct piezoelectric response (i.e., $d_{33}=d_{330}+\alpha^{T} T_{0}$ ), we can obtain the Rayleigh coefficient related to piezoelectric response, $\alpha^{T}$, by a linear fitting of the $d_{33}$ versus $T_{0}$ curves. The value $\alpha_{C S}^{T}=8.4(\mathrm{pC} / \mathrm{N}) / \mathrm{MPa}$ for PNZT-CS is almost four times higher than the value $\alpha_{S P S}^{T}=2.3(\mathrm{pC} / \mathrm{N}) / \mathrm{MPa}$ for PNZT-SPS, indicating an effective drop in the nonlinear behavior by grain-size reduction up to a submicron scale. Fig. 5(b) shows the value of the longitudinal piezoelectric coefficient at zero dynamical 
stress as a function of the applied bias stress. As may be observed, the value of $d_{330}$ for PNZT-CS is higher than the value for PNZT-SPS at low $T_{\mathrm{DC}}$, as is expected due to the major extrinsic response exhibited by the PNZT-CS sample. However, a drastic decrease in $d_{330}$ is displayed in PNZT-CS on increasing $T_{\mathrm{DC}}$ from $15 \mathrm{MPa}$ to $55 \mathrm{MPa}$, while $d_{330}$ remains practically unaltered (i.e., a decrease of $5 \%$ from the initial value on increasing $T_{\mathrm{DC}}$ from $20 \mathrm{MPa}$ to $60 \mathrm{MPa}$ ). Despite the higher piezoelectric coefficient of PNZT-CS compared to PNZT-SPS at low compression pre-stress, the $d_{330}$ values at $T_{\mathrm{DC}}$ above $40 \mathrm{MPa}$ are higher in PNZT-SPS than in PNZT-CS. This fact proves the enhancement of the linear piezoelectric response in submicron-structured piezoceramics at high pre-stress compression. Fig. 5(c) shows the variation of the Rayleigh coefficient with the applied bias stress. One may observe that $\alpha^{\mathrm{T}}$ is a constant value for PNZTSPS, which shows the stability of the direct piezoelectric response in the PNZT-SPS sample.

It is well known that the major contribution to the functional response of piezoceramics comes from the movement of non- $180^{\circ}$ domain walls [24]. Moreover, due to the fact that complex domain structures can be formed inside each grain [30], the movement of non- $180^{\circ}$ domain walls can be inhibited not only by the interaction with defects, but also by the interaction with grain boundaries, or even by domain coalescence. If the non- $180^{\circ}$ domain walls can move with little restriction over a relatively long range inside the grain, which is expected to occur in PNZT-CS, the action of a static compression may produce by coalescence a partial and reversible elimination of non- $180^{\circ}$ domains, thereby reducing the number of domain walls [29]. As a consequence, the extrinsic contribution reduces under compression, leading to a decrease in the $d_{330}$ coefficients. This effect can be explained if the interaction energy between the pinning centers and the domain walls is sufficiently low for the walls to overcome the local lattice defects and acquire a substantial motion needed for coalescence. It must be noted that, from a macroscopic viewpoint, this effect may be perceived as a clamping of domain walls. On the 
other hand, due to the lower grain size of the PNZT-SPS sample, the restriction of the domain wall movement due to the proximity of the grain boundaries must be also taken into account. Restricted domain wall motion inhibits the coalescence, thereby making the stabilization of the functional properties in submicron-structured piezoceramics possible for a wide range of static compression. Unquestionably, this is an important result from a practical point of view.

\section{Conclusions}

The nonlinear behavior of piezoceramics is a drawback that requires a solution for many applications. It is an issue that is usually tackled by compositional engineering, and to a lesser extent by microstructural engineering. In this work, we demonstrate that an effective control of grain size enhances properties stability in piezoceramics. Submicron-structured piezoceramics exhibit a high piezoelectric response stability when the materials work under a compression stress. The Rayleigh model is used to study the nonlinear response quantitatively. Results from the model indicate that the extrinsic contribution to functional properties in submicron-structured soft-PZT is governed by the irreversible movement of domain walls, as in the microstructured sample. Accordingly, the enhancement of properties stability appears to be due to a clamping effect by the proximity of the grain boundary.

\section{Acknowledgement}

F. R-M. thanks the Spanish Ministry of Economy and Competitiveness (MINECO) for a 'Ramon y Cajal' contract (Ref: RyC-2015-18626), which is co-financed by the European Social Fund. 
This is the post-print (i.e. final draft post-refereeing) of the publication.

The final publication is available at Elsevier via http://dx.doi.org/10.1016/j.jeurceramsoc.2018.06.016

\section{References}

[1] M. Algueró, T. Hungría, H. Amorín, J. Ricote, J. Galy, A. Castro, Relaxor behaviour, polarization build up, and switching in nanostructured 0.92 $\mathrm{PbZn}_{1 / 3} \mathrm{Nb}_{2 / 3} \mathrm{O}_{3}-0.08 \mathrm{PbTiO}_{3}$ ceramics, Small 3 (2007) 1906- 1911.

[2] M. Algueró, H. Amorín, T. Hungría, J. Galy, A. Castro, Macroscopic ferroelectricity and piezoelectricity in nanostructured BiScO3-PbTiO3 ceramics, Appl. Phys. Lett. 94 (2009) 012902.

[3] L. Chao, Y. Hou, M. Zheng, Y. Yue, M. Zhu, Macroscopic ferroelectricity and piezoelectricity in nanostructured $\mathrm{NaNbO}_{3}$ ceramics, Appl. Phys. Lett. 110 (2017) 122901.

[4] Z. A. Munir, U. Anselmi-Tamburini, M. Ohyanagi, The effect of electric field and pressure on the synthesis and consolidation of materials: A review of the spark plasma sintering method, J. Mater. Sci. 41 (2006) $763-777$.

[5] T. Hungría, J. Galy, A. Castro, Spark plasma sintering as a useful technique to the nanostructuration of piezoferroelectric materials, Adv. Eng. Mater. 11 (2009) 615-631.

[6] L. Mitoseriu, V. Tura, D. Ricinschi, C. Harnagea, Grain size dependence of the Rayleigh coefficients in barium titanate ceramics, Ferroelectrics 240 (2000) 1317-1324.

[7] M.T. Buscaglia, M. Viviani, V. Buscaglia, L. Mitoseriu, A. Testino, P. Nanni, Z. Zhao, M. Nygren, C. Harneaga, D. Piazza and C. Galassi, High dielectric constant and frozen macroscopic polarisation in dense nanocrystalline $\mathrm{BaTiO}_{3}$ ceramics, Phys. Rev. B 73 (2006) 064114.

[8] M. Algueró, J. Ricote, R. Jiménez, P. Ramos, J. Carreaud, B. Dkhil, J. M. Kiat, J. Holc, M. Kosec, Size effect in morphotropic phase boundary $\mathrm{Pb}\left(\mathrm{Mg}_{1 / 3} \mathrm{Nb}_{2 / 3}\right) \mathrm{O}_{3}-\mathrm{PbTiO}_{3}$, Appl. Phys. Lett. 91 (2007) 112905.

[9] H. Amorín, J. Ricote, R. Jiménez, J. Holc, M. Kosec, M. Algueró, Submicron and nanostructured $0.8 \mathrm{~Pb}\left(\mathrm{Mg}_{1 / 3} \mathrm{Nb}_{2 / 3}\right) \mathrm{O}_{3}-0.2 \mathrm{PbTiO}_{3}$ ceramics by hot pressing of nanocrystalline powders, Scripta Mater. 58 (2008) $755-758$.

[10] D. Ghosh, A. Sakata, J. Carter, P. A. Thomas, H. Han, J. C. Nino, J. L. Jones, Domain wall displacement is the origin of superior permittivity and piezoelectricity in $\mathrm{BaTiO}_{3}$ at intermediate grain sizes, Adv. Funct. Mater. 24 (2014) 885-896.

[11] Y. Tan, J. Zhang, Y. Wu, C. Wang, V. Koval, B. Shi, H. Ye, R. McKinnon, G. Viola, H. Yan, Unfolding grain size effects in barium titanate ferroelectric ceramics, Sci. Rep. 5 (2015) 9953.

[12] Q. M. Zhang, H. Wang, N. Kim, L. E. Cross, Direct evaluation of domain wall and intrinsic contributions to the dielectric and piezoelectric response and their temperature dependence on lead zirconate titanate, J. Appl. Phys. 75 (1994) 454-459.

[13] C.A. Randall, N. Kim, J.P. Kucera, W. Cao, T.R. Shrout, Intrinsic and extrinsic size effects in fine grained morphotropic phase boundary lead zirconate ceramics, J. Am. Ceram. Soc. 81 (1998) 677-688.

[14] J. L. Jones, M. Hoffman, J. E. Daniels, A. J. Studer, Direct measurement of the domain switching contribution to the dynamic piezoelectric response in ferroelectric ceramics, Appl. Phys. Lett. 89 (2006) 092901.

[15] D. A. Ochoa, J. E. Garcia, R. Perez, A. Albareda, Influence of extrinsic contribution on the macroscopic properties of hard and soft lead zirconate titanate ceramics, IEEE Trans. Ultrason. Ferroelectr. Freq. Control 55 (2008) 2732-2736. 
This is the post-print (i.e. final draft post-refereeing) of the publication.

The final publication is available at Elsevier via http://dx.doi.org/10.1016/j.jeurceramsoc.2018.06.016

[16] D. A. Ochoa, G. Esteves, T. Iamsasri, F. Rubio-Marcos, J. F. Fernández, J. E. Garcíaa, J. L. Jones, Extensive domain wall contribution to strain in a $(\mathrm{K}, \mathrm{Na}) \mathrm{NbO}_{3}$-based lead-free piezoceramics quantified from high energy X-ray diffraction, J. Eur. Ceram. Soc. 36 (2016) 2489-2494.

[17] J. E. Garcia, R. Pérez, A. Albareda, J.A. Eiras, Non-linear dielectric and piezoelectric response in undoped and $\mathrm{Nb}^{5+}$ or Fe ${ }^{3+}$ doped PZT ceramic system, J. Eur. Ceram. Soc. 27 (2007) 4029-4032.

[18] J. E. García, R. Pérez, D. A. Ochoa, A. Albareda, M. H. Lente, J. A. Eiras, Evaluation of domain wall motion in lead zirconate titanate ceramics by nonlinear response measurements, J. Appl. Phys. 103 (2008) 054108.

[19] J. E. García, Extrinsic contribution and instability properties in lead-based and lead-free piezoceramics, Materials 8 (2015) 7821-7836.

[20] D. Damjanovic, A morphotropic phase boundary system based on polarization rotation and polarization extension, Appl. Phys. Lett. 97 (2010) 062906.

[21] D. A. Ochoa, G. Esteves, J. L. Jones, F. Rubio-Marcos, J. F. Fernández, J. E. García, Extrinsic response enhancement at the polymorphic phase boundary in piezoelectric materials, Appl. Phys. Lett. 108 (2016) 142901.

[22] J. E. García, R. Pérez, A. Albareda, High electric field measurement of dielectric constant and losses of ferroelectric ceramics, J. Phys. D. Appl. Phys. 34 (2001) 3279-3284.

[23] A. Barzegar, D. Damjanovic, N. Setter, The effect of boundary conditions and sample aspect ratio on apparent $\mathrm{d}_{33}$ piezoelectric coefficient determined by direct quasi static method, IEEE Trans. Ultrason. Ferroelectr. Freq. Control 51 (2004) 262-270.

[24] D. Damjanovic, M. Demartin, Contribution of the irreversible displacement of domain walls to the piezoelectric effect in barium titanate and lead zirconate titanate ceramics, J. Phys. Condens. Matter 9 (1999) 4943-4953.

[25] J. E. Garcia, D. A. Ochoa, V. Gomis, J. A. Eiras, R. Pérez, Evidence of temperature dependent domain wall dynamics in hard lead zirconate titanate piezoceramics, J. Appl. Phys. 112 (2012) 014113.

[26] D. Damjanovic, M. Demartin, The Rayleigh law in piezoelectric ceramics, J. Phys. D. Appl. Phys. 29 (1999) 2057-2060.

[27] D. A. Hall, Nonlinearity in piezoelectric ceramics, J. Mater. Sci. 36 (2001) 4575-4601.

[28] S. Li, W. Cao, L. E. Cross, The extrinsic nature of nonlinear behavior observed in lead zirconate titanate ferroelectric ceramic, J. Appl. Phys. 69 (1991) 7219-7224.

[29] D. A. Ochoa, J. E. García, I. Tamayo, V. Gomis, D. Damjanovic, R. Perez, Effect of uniaxial compressive stress on dielectric and piezoelectric responses in lead zirconate titanate based ceramics, J. Am. Ceram. Soc. 95 (2012) 1656-1660.

[30] R. Pérez, J. E. García, A. Albareda, V. Gomis, D.A. Ochoa, Motion of $90^{\circ}$ and $180^{\circ}$ ferroelectric domain wall structures, Mech. Mater. 42 (2010) 374-382. 